\title{
Curva de crescimento de cordeiros oriundos de três sistemas de produção na Região Nordeste do Brasil
}

\section{Growth curve of lambs from three systems of production in Northeastern Brazil}

\author{
Marcílio Costa Teixeira $^{1 *}$; Arturo Bernardo Villarroel2; \\ Elzania Sales Pereira ${ }^{3}$; Sônia Maria Pinheiro de Oliveira ${ }^{4}$; \\ Ítalo Araújo Albuquerque ${ }^{5}$ Ivone Yurika Mizubuti ${ }^{6}$
}

\begin{abstract}
Resumo
O objetivo foi analisar modelos não lineares para descrever o crescimento de cordeiros criados em três Sistemas de produção. Os modelos utilizados forram: Brody, Von Bertalanffy, Richards e Gompertz. No Sistema de produção 1 utilizaram-se cordeiros alimentados em "creep-feeding", desmamados aos 60 dias de idade e mantidos em pastagem cultivada até atingirem media de $20 \mathrm{~kg}$, quando foram confinados, e abatidos entre 28 e $30 \mathrm{~kg}$. No Sistema de produção 2 utilizaram-se cordeiros desmamados aos 90 dias e mantidos em pastagem cultivada, sendo abatidos entre 28 e $30 \mathrm{~kg}$. No Sistema de produção 3 utilizaram-se cordeiros desmamados com 90 dias de idade e mantidos em área de pastagem nativa suplementados com feno de capim elefante e de leucena no período crítico do ano e abatidos aos 28 e $30 \mathrm{~kg}$. Foram utilizadas 17 pesagens de 30 animais, sendo 10 animais para cada Sistema, do nascimento aos 450 dias. Com base no estudo das curvas de crescimento, as que melhor se adaptaram para predição dos resultados de crescimento foram as de Richards e Brody, entretanto nenhum dos modelos de curva simulados apresentou resultados satisfatórios para o Sistema de produção 3.
\end{abstract}

Palavras-chave: Desempenho, produção, “creep-feeding”, pastagem cultivada, pastagem nativa, manejo

\begin{abstract}
The objective was to analyze nonlinear models to describe growth of lambs in three production systems. The models used were: Brody, Von Bertalanffy, Gompertz and Richards. In a 1 production system was used lambs in "creep feeding", weaned at 60 days old and kept on pasture until they reach average 20 $\mathrm{kg}$, when they were confined, slaughtered between 28 and $30 \mathrm{~kg}$. In the 2 production system, lambs weaned at 90 days and kept on cultivated pasture and slaughtered between 28 and $30 \mathrm{~kg}$, were used. In 3 Production System, lambs weaned at 90 days old and kept in an area of native pasture supplemented with elephant grass and leucena, in the critical period of the year and slaughtered at 28 to $30 \mathrm{~kg}$ were used. 17 weights of 30 animals, 10 animals for each system, from birth to 450 days, were used. Based on the study of the growth curves that are best adapted to predict the results of growth were those of Richards and Brody models, however none of the models simulated curve showed satisfactory results for the three production systems.
\end{abstract}

Key words: Performance, production, creep-feeding, pasture, native pasture

1 Prof. Dr. do Instituto Federal de Educação, Ciência e Tecnologia. Campus de Crateús, Crateús. CE. E-mail: marcilioct@hotmail.com

2 Prof. Dr. do Dept $^{0}$ de Zootecnia da Universidade Federal do Ceará, UFC, Av. Mister Hull, 2977, Fortaleza, CE. Tel. +55.85.33669749. Pesquisadores do CNPq. E-mail: selaive@ufc.br

3 Prof $^{\mathrm{a}} \mathrm{Dr}^{\mathrm{a}}$ do Dept ${ }^{\mathrm{o}}$ de Zootecnia da UFC, Fortaleza, CE. Pesquisadora do CNPq. E-mail: elzania@hotmail.com

4 Prof $^{\mathrm{a}} \mathrm{Dr}^{\mathrm{a}}$ do Dept ${ }^{\mathrm{o}}$ de Zootecnia da UFC, Fortaleza, CE. E-mail: soniace@ufc.br

5 Discente de Doutorado do Programa de Doutorado integrado em Zootecnia, UFC, Fortaleza, CE. E-mail: italozoo@hotmail.com

6 Prof $^{a}$. Dr ${ }^{\mathrm{a}}$. do Dept $^{\mathrm{o}}$ de Zootecnia da Universidade Estadual de Londrina, UEL, Londrina, PR. Pesquisadora do CNPq. E-mail: mizubuti@uel.br

* Autor para correspondência 


\section{Introdução}

$\mathrm{Na}$ criação de ovinos um dos objetivos mais importantes é obter cordeiros com peso adequado e alto rendimento de carcaça em curto período de tempo. As épocas alternativas de desmame e o efeito da suplementação de alimentos apresentam-se como caminho para o desenvolvimento de sistemas de produção de carne ovina nas condições peculiares do nordeste. Nesta região, a maioria dos cordeiros são desmamados ao redor de 120 a 126 dias de idade, permanecendo ao lado da ovelha em pastagem nativa que em geral, não oferece condições para que o animal apresente seu potencial produtivo. Como consequência, as crias desenvolvem-se lentamente.

O crescimento de cordeiros em pastagem é condicionado principalmente por dois fatores que atuam sobre seu potencial genético: nutrição e parasitismo. Após o nascimento, a nutrição de cordeiros depende da produção de leite da ovelha e da disponibilidade de forragem e suplementação alimentar, portanto, o crescimento do cordeiro pode ser melhorado por meio da nutrição, com suplementação da ovelha no inicio da lactação e do cordeiro a partir da $2^{\mathrm{a}}$ semana de idade.

As curvas de crescimento que relacionam o peso do animal com a idade utilizam modelos não lineares para condensar grande volume de informação em pequeno conjunto de parâmetros que podem ser interpretados biologicamente (McMANUS et al., 2003). O ajuste de funções de crescimento peso-idade é uma ferramenta fundamental no planejamento da produção animal, pois permite obter informações precisas sobre ganho de peso dos animais, além de informações de prognósticos futuros para animais do mesmo grupo racial sob a mesma situação ambiental.

Modelos não lineares que têm sido usados para ajustar a relação entre tamanho e idade dos animais, em geral, são referidos como função Richard, que é representativa de um número infinito de casos incluindo as funções Brody, Von Betalanffy, Gompertz e Logística (FITZHUGH JUNIOR, 1976).
O objetivo deste trabalho foi analisar quais modelos não lineares apresentam melhor ajuste a curva de crescimento de animais criados em três sistemas de produção, para melhor auxiliar na tomada de decisão e no manejo dos rebanhos e predição de resultados.

\section{Material e Métodos}

O experimento foi conduzido na Fazenda Experimental Vale do Curu, pertencente à Universidade Federal do Ceará, localizada no município de Pentecoste - CE, situada a $3^{\circ} 47^{\prime}$ latitude sul e $39^{\circ} 17^{\prime}$ longitude oeste e 78 metros de altitude em relação ao nível do mar.

O clima da região é classificado como tropical subúmido (tu), segundo classificação de Köppen, sendo identificadas duas épocas do ano em termos de precipitação pluvial: uma chuvosa, que se estende de janeiro a junho e outra seca, que se estende de julho a dezembro. A precipitação pluviométrica média foi de $809,8 \mathrm{~mm}$, sendo os meses de março e abril os mais chuvosos. A temperatura média foi de $27,28^{\circ} \mathrm{C}$ e umidade relativa de $64,41 \%$ (Estação Meteorológica daFEVC-UFC), sendo consideradas normais em relação a medias históricas.

A área experimental constou de três piquetes de pastagem nativa, caatinga rebaixada e raleada, com aproximadamente 14 ha cada, a área de pastagem cultivada formada de 0,54 ha de capim gramão (Cynodon dactylon, L.), 0,53 ha de capim corrente (Urochloa mosambicensis) e 0,98 ha de capim buffel (Cenchrus ciliares, L), além uma área de aproximadamente 0,73 ha de capineira de capim elefante (Pennicetum purpureum) e aproximadamente 1 ha de leucena (Leucaena leucocephala) para produção de feno.

Todos os animais utilizados nesse estudo eram da raça Santa Inês, que nasceram no mesmo período e de parto simples. No Sistema de produção 1 utilizaramse 10 cordeiros oriundos de ovelhas mantidas em pastagem cultivada durante a gestação e lactação e 
suplementados por meio de "creep-feeding" a partir da segunda semana de vida até o desmame, com 60 dias de idade, pesando em média 11,02 kg. Após o desmame os animais permaneceram em pastagem cultivada recebendo suplementação alimentar na forma de ração concentrada, $0,2 \mathrm{~kg} /$ animal/dia no inicio do confinamento e $0,4 \mathrm{~kg}$ no ultimo mês de acabamento, e sal mineral durante todo o período experimental. Após os animais atingirem peso médio de $20 \mathrm{~kg}$, foram confinados até os cordeiros atingirem o peso médio de abate de 28 a $30 \mathrm{~kg}$, com 180 dias de idade.

No Sistema de produção 2 utilizaram-se 10 cordeiros oriundos de ovelhas mantidas em pastagem cultivada durante a parição e lactação, desmamados aos 90 dias de idade, pesando em média 14,23 kg. Após o desmame, os cordeiros foram mantidos em pastagem cultivada, até atingirem o peso médio de abate de 28 a $30 \mathrm{~kg}$, com 390 dias de idade. Os animais receberam suplemento mineral durante todo o período experimental.

No Sistema de produção 3 utilizaram-se 10 cordeiros oriundos de ovelhas mantidas em pastagem nativa, caatinga raleada e rebaixada, durante todo o período experimental, desmamados aos 90 dias de idade, com peso médio de 13,56 $\mathrm{kg}$. Após o desmame, os cordeiros continuaram mantidos em pastagem nativa até o abate com peso médio de 28 a $30 \mathrm{~kg}$ e 450 dias de idade. Os animais receberam suplementação mineral durante todo período experimental e alimentação na forma de feno de capim elefante (Pennisetum purpureum) e leucena (Leucaena leucocephala) no período crítico da época seca (outubro, novembro e dezembro).

O desempenho dos animais foi avaliado por meio de pesagens a cada 15 dias do nascimento à desmama e a cada 30 dias da desmame ao abate dos machos.

Os dados de peso e ganho de peso foram agrupados em delineamento inteiramente casualizado e analisados utilizando-se o PROCGLM do pacote estatístico SAS (2009). Para as curvas de crescimento foi realizado analise de regressão para confecção das curvas, utilizando-se o procedimento PROC-REG do pacote estatístico SAS (2009).

Foram ajustados os modelos de Brody, Von Bertalanffy, Gompertz e Richards (Tabela 1) para estimar o crescimento dos animais e os parâmetros da curva.

Tabela 1. Forma geral dos modelos não lineares.

\begin{tabular}{lc}
\hline Modelo & Forma Geral \\
\hline Brody & $\mathrm{Y}=\mathrm{A}\left(1-\mathrm{Be}^{-\mathrm{kt}}\right)+\epsilon$ \\
Von Bertalanffy & $\mathrm{Y}=\mathrm{A}\left(1-\mathrm{Be}^{-k t}\right)^{3}+\epsilon$ \\
Richards & $\mathrm{Y}=\mathrm{A}\left(1-\mathrm{Be}^{-k t}\right)^{-\mathrm{m}}+\epsilon$ \\
Gompertz & $\mathrm{Y}=\mathrm{Ae}^{\mathrm{Be}(-\mathrm{kit})}+\epsilon$ \\
\hline
\end{tabular}

Fonte: Elaboração dos autores.

A função Richards descreve mudanças no tamanho Y (peso) em relação à idade $t$, sendo representada pela equação

$$
\mathrm{Yt}=\mathrm{A}(1 \pm \mathrm{Bekt}) \mathrm{m},
$$

onde:

Y: é o peso corporal à idade $t$;

A: é o valor assintótico, interpretado como peso assintótico ou medida do peso à maturidade;

B: o parâmetro escala (constante de integração), indicando a proporção do crescimento assintótico a ser ganho depois do nascimento, estabelecido pelos valores iniciais de $\mathrm{Y}$ e $\mathrm{t}$;

k: o índice de maturidade ou medida de precocidade. Fazendo-se k-1, obtém-se o intervalo de tempo gasto para atingir a maturidade, o que serve para medir as mudanças no grau de maturidade. Maior valor de $\mathrm{k}$ indica os animais que chegam à maturidade mais cedo;

m é o parâmetro de inflexão, que estabelece o grau de maturidade u no ponto de inflexão ui $=[(\mathrm{M}-1)$ $/ \mathrm{M}] \mathrm{M}$, sendo ut a proporção de peso atingido à idade t: 
ut $=\mathrm{Yt} / \mathrm{A}=(1-\mathrm{Be}-\mathrm{kt}) \mathrm{M}$;

$\in$ : é base de Napier para logaritmos naturais.

Os parâmetros tem o mesmo significado para as outras curvas. Foi utilizado o método GaussNewton no procedimento NLIN (SAS, 2009).

Os critérios utilizados para selecionar o modelo que melhor descreveu a curva de crescimento foram os mesmos utilizados por Carneiro et al. (2009): 1) quadrado médio do resíduo (QMR) - calculado dividindo-se a soma de quadrados do resíduo, pelo número de observações, que é estimador de máxima verossimilhança da variância residual, para que se pudesse comprar o QMR dos diferentes modelos, pelo fato de possuírem números diferentes de parâmetros a serem estimados; 2) coeficiente de determinação $\left(\mathrm{R}^{2}\right)$ - calculado como o quadrado da correlação entre os pesos observados e estimados, por meio de uma analise de regressão linear, considerou-se o peso predito pelo modelo como variável dependente e o peso observado do animal como variável independente; 3) analise gráfica do modelo com o peso médio observado; 4) desvio médio absoluto dos resíduos (DMA), estatística proposta por Sarmento et al. (2006) para avaliar a qualidade de ajuste, calculada como:

$$
D M A=\frac{\sum_{i=1}^{n}|Y-\hat{Y} i|}{n}
$$

Onde, $\mathrm{Y}_{\mathrm{i}}$ é o valor observado, $\hat{Y}$ o valor estimado e n o tamanho da amostra. Quanto menor o valor de DMA, melhor o ajuste aos resultados reais.

\section{Resultados e Discussão}

Os pesos e desvios padrões e as estimativas dos parâmetros para cada modelo de crescimento e os critérios utilizados para avaliar quais desses modelos melhor se ajustam às curvas de crescimento de cordeiros mantidos em três diferentes Sistemas de produção encontram-se nas Tabelas 2, 3, 4.

Tabela 2. Média e desvio padrão de pesos observados ao nascimento (PN) e ajustados aos 30 (P30), 60 (P60), 90 (P90), 120 (P120), 150 (P150), 180 (P180), 210 (P210), 240 (P240), 270 (P270), 300 (P300), 330 (P330), 360 (P360), 390 (P390), 420 (P420) e 450 (P450) dias de idade em ovinos Santa Inês criados em três Sistemas de produção.

\begin{tabular}{lccc}
\hline \multirow{2}{*}{ Pesos $(\mathrm{kg})$} & \multicolumn{3}{c}{ Média de Pesos $(\mathrm{kg})$ e Desvio Padrão $(\mathrm{kg})$} \\
\cline { 2 - 4 } & Sistema 1 & Sistema 2 & Sistema 3 \\
\hline PN & $2,9 \pm 0,66$ & $3,0 \pm 0,20$ & $3,23 \pm 0,36$ \\
P30 & $6,93 \pm 0,43$ & $8,59 \pm 1,38$ & $7,63 \pm 0,36$ \\
P60 & $11,02 \pm 0,40$ & $11,73 \pm 0,36$ & $11,41 \pm 0,46$ \\
P90 & $19,85 \pm 2,74$ & $14,23 \pm 0,36$ & $13,56 \pm 0,70$ \\
P120 & $25,61 \pm 3,82$ & $17,06 \pm 0,80$ & $16,48 \pm 0,79$ \\
P150 & $27,16 \pm 3,51$ & $20,22 \pm 1,20$ & $19,38 \pm 0,98$ \\
P180 & $30,61 \pm 0,40$ & $23,35 \pm 2,16$ & $22,74 \pm 1,65$ \\
P210 & - & $23,89 \pm 2,01$ & $23,44 \pm 1,72$ \\
P240 & - & $24,85 \pm 2,07$ & $24,38 \pm 1,78$ \\
P270 & - & $25,89 \pm 1,84$ & $25,11 \pm 1,84$ \\
P300 & - & $26,97 \pm 1,84$ & $25,39 \pm 2,09$ \\
P330 & - & $27,49 \pm 1,80$ & $25,85 \pm 2,12$ \\
P360 & - & $27,78 \pm 1,73$ & $26,35 \pm 2,14$ \\
P390 & - & $28,89 \pm 0,85$ & $27,00 \pm 2,21$ \\
P420 & - & - & $27,63 \pm 2,14$ \\
P450 & - & - & $28,58 \pm 1,70$ \\
\hline
\end{tabular}

Fonte: Elaboração dos autores. 
Tabela 3. Estimativas dos parâmetros (A, B, k e m), quadrado médio do resíduo (QMR), coeficiente de determinação $\left(\mathrm{R}^{2}\right)$, percentual de convergência $(\mathrm{C})$ e desvio médio absoluto (DMA) para o Sistema 1 de produção de acordo com os modelos estudados.

\begin{tabular}{lcccccccc}
\hline \multirow{2}{*}{ Modelo } & \multicolumn{9}{c}{ Parâmetros } & \multirow{2}{*}{ QMR } & \multirow{2}{*}{$\mathrm{R}^{2}$} & \multirow{2}{*}{$\mathrm{C}(\%)$} & \multirow{2}{*}{ DMA } \\
\cline { 2 - 4 } & $\mathrm{A}(\mathrm{kg})$ & $\mathrm{B}$ & $\mathrm{k}$ & $\mathrm{m}$ & & & & \\
\hline Brody & 30,08 & 0,92 & 0,006 & - & 8,60 & 99,87 & 100 & 0,79 \\
Von Bertalanffy & 28,12 & 0,50 & 0,009 & - & 8,72 & 98,04 & 100 & 0,53 \\
Richards & 30,08 & 0,92 & 0,001 & 5,5 & 8,60 & 87,09 & 100 & 0,52 \\
Gompertz & 27,61 & $-1,95$ & 0,011 & - & 8,89 & 98,01 & 100 & 0,51 \\
\hline
\end{tabular}

Fonte: Elaboração dos autores.

Tabela 4. Estimativas dos parâmetros (A, B, k e m), quadrado médio do resíduo (QMR), coeficiente de determinação $\left(\mathrm{R}^{2}\right)$, percentual de convergência $(\mathrm{C})$ e desvio médio absoluto (DMA) para o Sistema 2 de produção de acordo com os modelos estudados.

\begin{tabular}{lcccccccc}
\hline \multirow{2}{*}{ Modelos } & \multicolumn{9}{c}{ Parâmetros } & \multirow{2}{*}{ QMR } & \multirow{2}{*}{$\mathrm{R}^{2}$} & \multirow{2}{*}{$\mathrm{C}(\%)$} & \multirow{2}{*}{ DMA } \\
\cline { 2 - 4 } Brody & $\mathrm{A}(\mathrm{kg})$ & $\mathrm{B}$ & $\mathrm{k}$ & $\mathrm{m}$ & & & \\
Von Bertalanffy & 31,30 & 0,90 & 0,006 & - & 2,21 & 96,65 & 100 & 0,58 \\
Richards & 29,80 & 0,48 & 0,009 & - & 2,28 & 99,56 & 100 & 0,71 \\
Gompertz & 31,30 & 0,90 & 0,006 & 1,00 & 2,21 & 96,65 & 100 & 0,65 \\
\hline
\end{tabular}

Fonte: Elaboração dos autores.

Tabela 5. Estimativas dos parâmetros (A, B, k e m), quadrado médio do resíduo (QMR), coeficiente de determinação $\left(\mathrm{R}^{2}\right)$, percentual de convergência $(\mathrm{C})$ e desvio médio absoluto (DMA) para o Sistema 3 de produção de acordo com os modelos estudados.

\begin{tabular}{lcccccccc}
\hline \multirow{2}{*}{ Modelos } & \multicolumn{9}{c}{ Parâmetros } & \multirow{2}{*}{ QMR } & \multirow{2}{*}{$\mathrm{R}^{2}$} & \multirow{2}{*}{$\mathrm{C}(\%)$} & \multirow{2}{*}{ DMA } \\
\cline { 2 - 4 } \cline { 2 - 4 } Brody & $\mathrm{A}(\mathrm{kg})$ & $\mathrm{B}$ & $\mathrm{k}$ & $\mathrm{m}$ & & & & \\
Von Bertalanffy & 14,40 & 0,82 & 0,027 & - & 0,93 & 94,47 & 100 & 0,84 \\
Richards & 13,70 & 0,41 & 0,040 & - & 1,00 & 99,02 & 100 & 1,32 \\
Gompertz & 14,40 & 0,82 & 0,013 & 2,0 & 0,93 & 94,47 & 100 & 0,79 \\
\hline
\end{tabular}

Fonte: Elaboração dos autores.

Pode-se verificar que para o sistema de produção 1,2 e 3 (Tabela 3, 4 e 5, respectivamente), as estimativas dos pesos (A), obtidos pelos quatro modelos, os maiores valores foram apresentados nos modelos de Brody e Richards seguidos por Von Bertalanffy e Gompertz (Figuras 1, 2 e 3).

Com base nos parâmetros (A, k, QMR, R², DMA) dos modelos de curva de crescimento para o Sistema de produção 1 e 2, o modelo de Brody foi o que melhor representou o crescimento dos animais desses Sistemas de produção.
Já para o Sistema de produção 3 com base nos mesmos parâmetros dos modelos de curvas de crescimento estudados, observou-se que nenhum dos modelos representou de maneira adequada o crescimento dos animais, principalmente em função do parâmetro A, que apresentou valor muito abaixo dos observados nos demais modelos de curva de crescimento estudados (Brody, Von Bertalanffy, Richards e Gompertz).

O parâmetro A apresenta uma estimativa do peso assintótico, que é interpretado como peso 
adulto (CARNEIRO et al., 2009). Esse peso não é o máximo que o animal atinge, e sim o peso médio à maturidade, livre das variações sazonais (BROWN; FITZHUGH JUNIOR; CARTWRIGHT, 1976). Existem controvérsias sobre o peso adulto ótimo, o qual depende da espécie, raça, seleção praticada previamente, do sistema de manejo e das condições climáticas (CARNEIRO et al., 2009). Esse parâmetro indica o peso que o animal atingirá à maturidade e que é útil na predição de resultados e planejamento da atividade como um todo.

Figura 1. Estimativa de peso em função da idade, obtidos pelos modelos não lineares de Brody, Von Bertalanffy, Gompertz e Richards em cordeiros criados no sistema de produção 1.

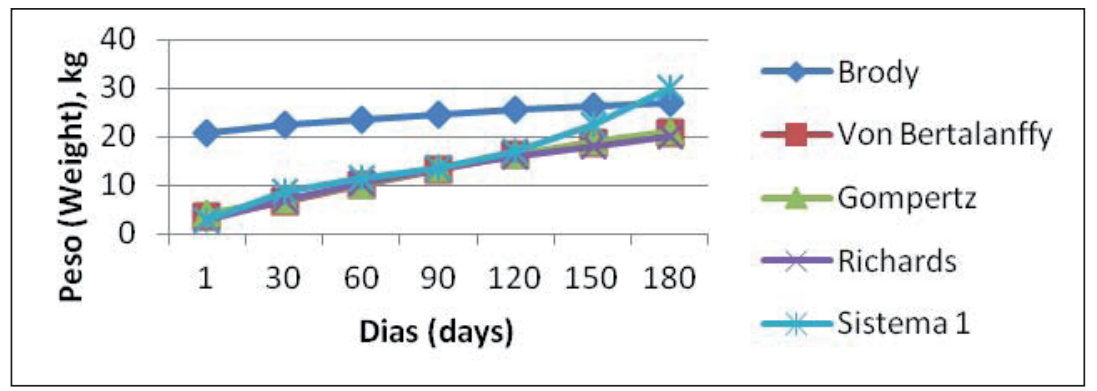

Fonte: Elaboração dos autores.

Figura 2. Estimativa de peso em função da idade, obtidos pelos modelos não lineares de Brody, Von Bertalanffy, Gompertz e Richards em cordeiros criados no sistema de produção 2.

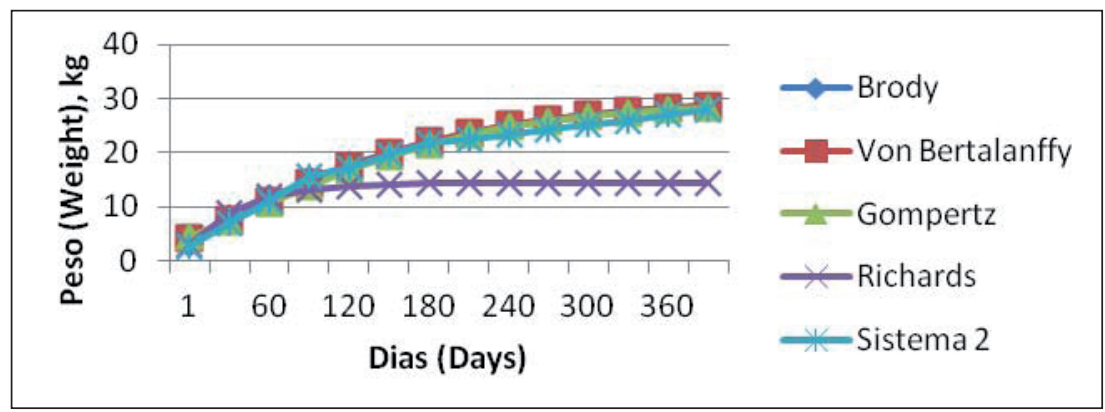

Fonte: Elaboração dos autores.

Figura 3. Estimativa de peso em função da idade, obtidos pelos modelos não lineares de Brody, Von Bertalanffy, Gompertz e Richards em cordeiros criados no sistema de produção 3.

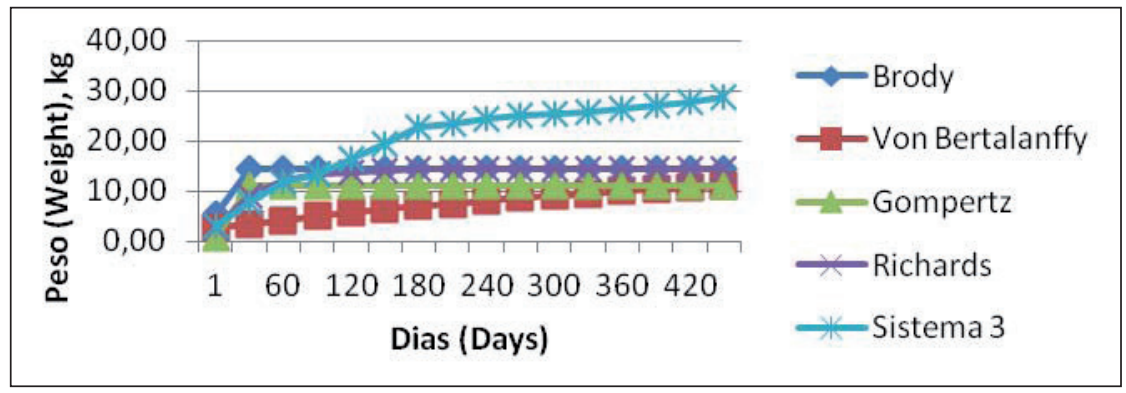

Fonte: Elaboração dos autores. 
Animais com alto valor de $\mathrm{k}$ são mais precoces, em comparação com aqueles de valores menores (CARNEIRO et al., 2009). O peso ao nascer dos animais não apresentou grande variação (Tabela 2), e em função disso os valores de k representaram bem a velocidade de crescimento desses animais. Segundo Oliveira, Lobo e Pereira (2000), para pesos iniciais similares, os valores de $\mathrm{k}$ representam, adequadamente as variações na velocidade de crescimento dos animais.

Com base nos coeficientes de determinação $\left(\mathrm{R}^{2}\right)$ (Tabelas 3, 4 e 5), observou-se que todos os modelos de curva de crescimento tiveram bons ajustes, à exceção dos modelos de curva de crescimento de Richards no Sistema de produção 1 e o modelo de crescimento de Gompertz, no Sistema de produção 3. O percentual de convergência (C) foi semelhante, no entanto na análise, o programa mostrou haver problemas com os modelos de Richards e Gompertz, o que pode ser um indicativo de que tais modelos de curva de crescimento não sejam adequados para predição de crescimento dos Sistemas de produção estudados nesse trabalho. Souza e Bianchini Sobrinho (1994) relataram que, quanto maior o número de convergências individuais em relação ao total de animais, melhor é o modelo. No entanto, não se podem desconsiderar os outros parâmetros para escolha do melhor modelo para predição de crescimento.

O desvio médio absoluto (DMA) indica a qualidade do ajuste das curvas de crescimento, pois quanto menor seu valor, melhor o ajuste. No entanto, não se pode considerar esse parâmetro isoladamente. Há necessidade de avaliar todos os parâmetros de forma conjunta para melhor decidir qual melhor modelo de curva de crescimento se aplica à determinado modelo de produção. Com base nisso, o que se observa é que para cada Sistema de produção houve um modelo de curva de crescimento que melhor se ajustou como foi observado nos outros parâmetros estudados, apresentados nas tabelas 3, 4 e 5 .
Braccini Neto et al. (1996) e Sarmento et al. (2006) relataram dificuldades de convergência com os modelos Richards e evidenciaram que, apesar da maior flexibilidade, por não fixar o ponto de inflexão, esse modelo apresenta maiores dificuldades no processo interativo em seu ajustamento.

Mazzini et al (2003) observaram que, de modo geral, para as duas configurações de modelos estudadas, as funções Logística, Gompertz e Von Bertalanffy, respectivamente, apresentaram maiores percentuais de convergência. Observouse ainda, tendência no aumento do percentual de convergência, nas funções de Gompertz e Von Bertalanffy, quando se utilizou a ponderação. Esse resultado está de acordo com Oliveira, Lobo e Pereira (2000), os quais, em trabalho com curvas de crescimento de fêmeas Guzerá, obtiveram maiores percentuais de convergência para essas funções e menores para as de Brody e Richards.

Elias (1998) observou aumento da convergência em todas as funções com a utilização da ponderação pelo inverso da variância, entretanto, vários autores alertaram sobre a dificuldade de convergência na função de Richards (BROWN; FITZHUGH JUNIOR; CARTWRIGHT, 1976; FITZHUGH JUNIOR, 1976; DENISE; BRINKS, 1985; PEROTTO; CUE; LEE, 1992).

\section{Conclusão}

Com base nos dados obtidos pode-se concluir que o desempenho dos cordeiros mantidos no Sistema 1 foram mais satisfatório por apresentar peso e ganho de peso superior aos dos outros Sistemas.

Com base no estudo das curvas de crescimento, as que melhor se adaptaram para predição dos resultados de crescimento foram as de Richards e Brody, mas nenhum dos modelos de curva simulados apresentaram resultados satisfatórios para o Sistema 3. 


\section{Referências}

BRACCINI NETO, J.; DIONELLO, N. J. L.; SILVEIRA JUNIOR, P.; BONGALHARDO, D. C.; XAVIER. E. G. Analise de curvas de crescimento de aves de postura. Revista Brasileira de Zootecnia, Viçosa, MG, v. 25, n. 6, p. 1062-1073, 1996.

BROWN, J. E.; FITZHUGH JUNIOR, H. A.; CARTWRIGHT, T. C. A comparison of nonlinear models for describing weight-age relationships in cattle. Journal of Animal Science, Champaign, v. 42, n. 4, p. 810-818, apr. 1976.

CARNEIRO, P. L. S.; MALHADO, C. H. M.; AFFONSO, P. R. A. M.; PEREIRA, D. G.; SUZART, J. C. C.; RIBEIRO JUNIOR, M.; ROCHA, J. L. Curva de crescimento em caprinos, da raça Mambrina, criados na caatinga. Revista Brasileira de Saúde e Produção Animal, Salvador, v. 10, n. 3, p. 536-545, 2009.

DENISE, R. S. K.; BRINKS, J. S. Genetic and environmental aspects of the growth curve parameters in beef cows. Journal of Animal Science, Champaign, v. 61, n. 6, p. 1431-1440, july 1985 .

ELIAS, A. M. Análise de curvas de crescimento de vacas das Raças Nelore, Guzerá e Gir. 1998. Dissertação (Mestrado em Ciência Animal e Pastagens) - Escola Superior de Agricultura Luiz de Queiroz, Piracicaba.

FITZHUGH JUNIOR, H. A. Analysis of growth curves and strategies for altering their shapes. Journal of Animal Science, Champaign, v. 42, n. 4, p. 1036-1051, apr. 1976.
MAZZINI, A. R. A.; MUNIZ, J. A.; AQUINO, L. H.; SILVA, F. F. Análise da curva de crescimento de machos hereford. Ciência Agrotécnica, Lavras. v. 27, n. 5, p. 1105-1112, 2003.

Mc MANUS, C.; EVANGELISTA, C.; FERNANDES, L. A. C.; MIRANDA, R. M.; MORENO-BERNAL, F. E.; SANTOS, N. R. Curva de crescimento de ovinos Bergamácia criados no Distrito Federal. Revista Brasileira de Zootecnia, Viçosa, MG, v. 32, n. 5, p. 12071212, 2003.

OLIVEIRA, H. N.; LÔBO, R. B.; PEREIRA, C. S. Comparação de modelos não lineares para descrever o crescimento de fêmeas da raça Guzerá. Pesquisa Agropecuária Brasileira, Brasília, v. 35, n. 9, p. 18431851, set. 2000.

PEROTTO, D.; CUE, R. I.; LEE, A. J. Comparison of nonlinear functions for describing the growth curve of three genotypes of dairy cattle. Canadian Journal of Animal Science, Ottawa, v. 72, n. 4, p. 773-782, dec. 1992.

SARMENTO, J. L. R.; REGAZZI, A. J.; SOUSA, W. H.; TORRES, R. A.; BREDA, F. C.; MENEZES, G. R. O. Estudo da curva de crescimento de ovinos Santa Inês. Revista Brasileira de Zootecnia, Viçosa, MG, v. 35, n. 2, p. 435-442, 2006.

SAS INSTITUTE. Statistical analyses sistem: user guide: Cary Stat. Verson 9.1. 2009.

SOUZA, J. C.; BIANCHINI SOBRINHO, E. Estimativas do peso de bovinos de corte, aos 24 meses, da raça Nelore usando curvas de crescimento. Revista Brasileira de Zootecnia, Viçosa, MG, v.23, n. 1, p. 85-91, 1994. 